American Journal of Infectious Diseases 7 (4): 83-90, 2011

ISSN 1553-6203

C 2011 Science Publications

\title{
Protective Effect of Enicostemma littorale Blume Methanolic Extract on Gentamicin-Induced Nephrotoxicity in Rats
}

\author{
${ }^{1,2}$ Niraj Mukundray Bhatt, ${ }^{1}$ Kinjal Chauhan, ${ }^{3}$ Sharad Gupta, \\ ${ }^{1}$ Prakash Pillai, ${ }^{4}$ Chirayu Pandya, ${ }^{5}$ Jyoti V. Thaikoottathil and ${ }^{1}$ Sarita S. Gupta \\ ${ }^{1}$ Department of Biochemistry, Faculty of Science, \\ M.S. University of Baroda, Vadodara, Gujarat 390002, India \\ ${ }^{2}$ Johns Hopkins University, School of Medicine \\ 720 Rutland Ave, 1054 Ross Bldg Baltimore MD 21205 \\ ${ }^{3}$ Gupta Pathology Laboratory, Dandia Bazar, \\ Vadodara, Gujarat 390001, India \\ ${ }^{4}$ Department of Psychiatry and Health Behavior, \\ Medical College of Georgia and Medical Research Service, \\ Veteran affairs Medical Center, 1 Freedom Way, Augusta, Georgia 30904, USA \\ ${ }^{5}$ Department of Medicine, National Jewish Health Center, 1400 Jackson St.,Denver, CO 80206, USA
}

\begin{abstract}
Problem statement: The Amino glycoside antibiotics cause drug induced nephrotoxicity at therapeutic doses and thus limits the use clinically. Oxidative stress is one of the main reasons for the development of nephrotoxicity. Approach: In light of this the aim of the present study was to investigate the role of mitochondrial as well as post-mitochondrial oxidative stress in the development of Gentamicin (GM) induced nephrotoxicity and to elucidate the role of Enicostemma Littoral blame (EL) extract, as a promising antioxidant therapy. Rats were divided into four groups, 1) (Control 2) GM (i.p., $80 \mathrm{mg} \mathrm{kg}^{-1}$ day $^{-1}$ for 8 days) (treated 3) GM+EL treated (i.g, $2.5 \mathrm{gm} \mathrm{kg}^{-1} \mathrm{day}^{-1}$ ) and (4) GM+ Vitamin C (VC) treated (i.g, $600 \mathrm{mg} \mathrm{kg}^{-1} \mathrm{day}^{-1}$ ). GM treated animals showed high oxidative stress in mitochondrial as well as post-mitochondrial fractions of renal tissues as evidenced by increased lipid per oxidation levels, decrease in GSH content and activities of antioxidant enzymes, SOD and GPx. Results: Oxidative stress was more pronounced in mitochondrial fraction as compared to post-mitochondrial fraction. GM-induced nephrotoxicity was further corroborated by an increase in serum cretonne and Blood Urea Nitrogen (BUN) levels and altered kidney histopathological observations. Treatment with EL ameliorates antioxidant defense system of mitochondrial as well as post-mitochondrial fraction, with better improvement seen in mitochondrial fraction. Conclusion: The present study explored beneficial effect of EL extract as an antioxidant therapy to counteract mitochondrial and post-mitochondrial oxidative stress generated in kidney upon GM-treatment, thus prevented nephrotoxicity.
\end{abstract}

Key words: Enicostemma littoral blame, oxidative stress, mitochondrial fraction, post-mitochondrial, Amino glycoside antibiotics, antioxidant therapy, Vitamin C (VC), lipid per oxidation

\section{INTRODUCTION}

Amino glycoside antibiotics, especially Gentamicin (GM) are widely applied in veterinary and human clinical practices for treatment of lifethreatening gram negative infections. These Aminoglycoside antibiotics also cause drug induced nephrotoxicity in $10-20 \%$ of therapeutic courses. Therefore, the clinical use of these drugs is limited by the development of nephrotoxicity. GM induced nephrotoxicity is characterized by tubular necrosis, without any morphological change in glomerular structures (Eisenberg et al., 1987; Cuzzocrea et al., 2002; Pedraza-Chaverri et al., 2003). Rats with GM-

Corresponding Author: Sarita Gupta, Department of Biochemistry, Faculty of Science, M.S. University of Baroda,

Vadodara, Gujarat 390002, India Tel: +91 2652795594 
induced nephrotoxicity provides an excellent model of acute renal failure and to test the protective effects of chemical compounds, plant extracts or drugs GMinduced nephrotoxicity is an ideal model (Ali and Bashir, 1996). The detailed mechanism by which GMinduces nephrotoxicity is not well understood; however, reactive oxygen species have been shown to be involved in pathogenesis (Cuzzocrea et al., 2002; Walker and Shah, 1988; Mazzon et al., 2001).

GM has been shown to generate superoxide anion $\left(\mathrm{O}_{2}\right)$, Hydrogen Peroxide $\left(\mathrm{H}_{2} \mathrm{O}_{2}\right)$ and Hydroxyl radical (HO) production from renal mitochondria (Yang et al., 1995; Walker et al., 1999). In addition to generation of free radicals it also increases (Guidet and Shah, 1989) lipoperoxidation (Pedraza-Chaverri et al., 2000; Ali, 2002), protein nitrosylation and carbonyaltion (Maldonado et al., 2003; Mazzon et al., 2001) and reduces glutathione content (Sener et al., 2002) in renal cortex from GM-treated rats. Morever, the administration of several compounds with antioxidant properties, Reactive Oxygen Species (ROS) scavengers and/or antioxidant enzymes were able to ameliorate the severity of GM-induced renal damage by reducing oxidative stress (Pedraza-Chaverri et al., 2000; Ali, 2002; Maldonado et al., 2003; Sener et al., 2002). In addition, kidney is deficient in the antioxidant enzymes Mn-Superoxide Dismutase (Mn-SOD) (PedrazaChaverri et al., 2000; Al-Majed et al., 2002) Glutathione Peroxidase (GPx) (Pedraza-Chaverri et al., 2000), Glutathione Reductase (GR) (Maldonado et al., 2003) and catalase (Pedraza-Chaverri et al., 2000) and thus more vulnerable to ROS in GM-treated rats.

Plants with potent antioxidant activity, has been shown to protect against gentamicin-induced nephrotoxicity. The Enicostemma littorale Blume is a small perennial herb of family Gentianaceae. The whole plant is used for medicinal purposes. It is commonly known as "chota chirayita" in Hindi or "mamejua" in Gujarati. We have shown hypoglycemic and antioxidant effect of aqueous as well as metabolic extract in alloxan-induced diabetic rats (Vijayvargia $e t$ al., 2000; Maroo et al., 2002; 2003a; 2003b) and also elucidated mechanism of its action (Maroo et al., 2002; 2003a). We are the first one to report the hypoglycemic, antioxidant and hypolipidemic properties of the aqueous extract of EL in newly diagnosed NIDDM patients (Vasu et al., 2003) as well as in cholesterol fed rats (Vasu et al., 2005). We have also demonstrated preventive effect of EL on nerve function and oxidative stress in animal model of diabetic neuropathy Bhatt $e t$ al. (2009) and islet neogenetic potential in vitro by purified compound from EL (Gupta et al., 2010). Apart from antidiabetic potential, we also exhibited vitro antimalarial activity of methanolic extract of plant and swertiamarin isolated from it (Soni and Gupta, 2009).

This herb is also known for its anti-inflammatory (Sadique et al., 1987; Roy et al., 2010), antiulcer (Roy et al., 2010) and anticancer property (Kavimani and Manisenthlkumar, 2000). The hepatoprotective effect of EL extract was examined in liver injury by carbon tetrachloride $\left(\mathrm{CCl}_{4}\right)$ in male Wistar rats (Gupta and Singh, 2007) as well as in ethanol-induced liver injury rat model (Baranisrinivasan et al., 2009). Hypolipidemic and hepatoprotective effect of aerial part of EL has been also evaluated in pdimethylaminobenzene (p-DAB) induced hepatotoxic animals (Gopal et al., 2004). Aqueous extract of EL has also shown effect on key carbohydrate metabolic enzymes in alloxan-induced diabetic rats (Srinivasan et al., 2005) and in vitro antioxidant activity (Vaijanathappa and Badami, 2009). C-glycosidic flavonoid derived from Enicostemma hyssopifolium showed aldose reductase inhibitory activity (Patel and Mishra, 2009). It is also used as an antidiabetic herbomineral preparation (Babu and Prince, 2004).

Swertiamarin is a secoiridoid glycoside present in EL, has been reported for its number of activity namely, hepatoprotective, antiedematogenic, free radical scavenging activity, antispastic activity (Vaijanathappa and Badami, 2009) and antinociceptive activity (Jaishree et al., 2008). Swertiamarin also showed antihyperlipidaemic effects in P-407-induced hyperlipidaemic rats (Vaidya et al., 2009a; 2009b) and as well as in cholesterol fed rats. Sonawane et al. (2010) reported ameliorative effect of swertiamarin and aqueous extract on nephropathic condition on type 1 diabetic animals. In the light of this, we have explored the possible protective role of Enicostemma littorale Blume, having good antioxidant potential (Maroo et al., 2003b) on gentamicin-induced oxidative stress in kidney, causing nepherotoxicity.

\section{MATERIALS AND METHODS}

Chemicals: All chemicals used were of analytical grade.

Extract preparation: Authentic plant material was purchased from local market and identified at the Botany Department, M.S. University, Baroda, Voucher specimen (Oza 51,51 (a)) is present at the Herbarium of Botany Department, M.S. University, Baroda. Whole dried plant was used for extract preparation. The plant 
was powdered in grinding meal. The powder was extracted with petroleum ether for $12 \mathrm{~h}$ in a Soxhlet apparatus. Residues were again extracted with methanol for $24 \mathrm{~h}$. After the extraction, methanol was recovered by distillation and remaining traces of methanol were completely removed by keeping methanol extract at $60^{\circ} \mathrm{C}$ for 4 days. The yield was found to be $40 \%$ and carboxymethylcellulose solution was used as vehicle (Maroo et al., 2003a).

Animals and treatment: Male Charles Foster rats (body weight 200-250 g) were used for the study. They were allowed ad libitum access to water and food. During the study, rats were maintained in stainless steel metabolic cages with a 12-h light/dark cycle to collect $24 \mathrm{~h}$ urine at the end of the study. All the animal studies were approved by Committee for the Purpose of Control and Supervision of Experiments on Animals (CPCSEA).

Rats were divided into four groups of six rats each as follows:

Group 1: Control (C), injected intraperitonially (i.p) with saline and intragastrically (i.g.) with $1 \%$ Carboxy Methyl Cellulose (CMC).

Group 2: Gentamicin Treated (GM) treated i.p with $\mathrm{GM}$ and i.g. with $1 \% \mathrm{CMC}$.

Group 3: $\quad$ GM + EL treated with EL and with GM. EL were given i.g. at a dose of $2.5 \mathrm{~g}$ of methanol extract $/ \mathrm{kg}$ body wt day ${ }^{-1}$ (Maroo et al., 2003b).

Group 4: $\mathrm{GM}+$ Vit $\mathrm{C}$ treated with Vit $\mathrm{C}$ and with GM. Vitamin $\mathrm{C}$ was given i.g at a dose of $600 \mathrm{mg} \mathrm{kg}^{-1}$ b.wt day ${ }^{-1}$.

The onset of damaging renal function induced by GM occurs after 5-8 days' treatments between of 80 and $150 \mathrm{mg} \mathrm{kg}^{-1}$. In this study, GM was injected intraperitoneally at the dose of $80 \mathrm{mg} \mathrm{kg}^{-1}$, for eight successive days, which is well known to cause significant nephrotoxicity in rats (Abdel-Gayoum et al., 1995; Kumar et al., 2000; Ali et al., 2004). The Enicostemma littorale extract was given at a dose of 2.5 $\mathrm{g} \mathrm{kg}^{-1}$ b. wt day ${ }^{-1}$ via gastric intubation for 11 days (Maroo et al., 2003a). The Vit C, potent antioxidant, was used as reference drug at a dose of $600 \mathrm{mg} \mathrm{kg}^{-1}$ b.wt day ${ }^{-1}$ (Kavutcu et al., 1996) for 11 days. EL and Vit $\mathrm{C}$ treatment was started three days prior to the 8 days gentamicin treatment and continued till the end of gentamicin treatment. At the end of experiment final weight of all animals were taken and urine collection has been done for $24 \mathrm{~h}$ with the help of metabolic cages. Blood samples were collected at the end of treatment on 9th day. The animals were sacrificed, kidney dissected out for estimation of various parameters as mentioned below. Change in body weight was monitored before and after the treatment.

Blood/serum creatinine and urea determination: Blood/serum creatinine (Reckon diagnostics Itd. CRK.008) and urea (Reckon diagnostics ltd. UAG.19N) were measured as the marker of glomerular damage at the end of the experiment. Blood Urea Nitrogen (BUN) was obtained by correcting the urea value by a 0.467 factor.

Antioxidant parameters: The tissues were minced and washed repeatedly with the sucrose buffer $\mathrm{pH}$ 7.4 to remove adhering blood and $10 \%(\mathrm{w} / \mathrm{v})$ homogenates were prepared using a Potter-Elvehjem type glass-Teflon homogenizer. Then the mitochondrial and post-mitochondrial fractions were prepared (Kaushal et al., 1999).

Lipid peroxides reacts with Thiobarbituric Acid (TBA) and forms Thiobarbituric Acid Reacting Substances) (TBARS) giving a characteristic pink colour which can be measured colorimetrically at 532 $\mathrm{nm}$ indicating the levels of LPO (Beuge and Aust, 1978). GSH levels were estimated by monitoring the reduction of DTNB (dithiobis-2-nitrobenzoic acid) forming a yellow coloured anion at $412 \mathrm{~nm}$ (Beutler $e t$ al., 1963). Measurements were carried out to determine the activities of Super Oxide Dismutase (SOD) (Marklund and Marklund, 1974; Patel and Katyare, 2006), Catalase (CAT) (Satav et al., 2000; Abei, 1984), Glutathione Peroxidase (GPx) (Paglia and Valentine, 1967). Protein estimation was according to the method of Lowry et al. (1951) using bovine serum albumin as the standard.

Histological analysis: Thin slices of kidney tissue with cortex and medulla were fixed by immersion in buffered formalin ( $\mathrm{pH} 7.4$ ), dehydrated and embedded in paraffin. Sections $(5 \mu \mathrm{m})$ were stained with hematoxilin and eosin (H and E). Light microscopy was used to evaluate tubular necrosis, which was graded as follows (Karahan et al., 2005).

Statistical analyses: Statistical analyses of data was done by Student's test, one-way analysis of variance and all groups were compared by means of Bonferroni, with significance set at $p<0.05$. All values represent the mean \pm S.E.M. Data were analyzed using PRISM Graph Pad software (version 3).

\section{RESULTS}

Body weight and urinary volume: Body weight among different treated group did not change significantly from the control group (Table 1). Urinary volume increased significantly in the GM group (Table 
1) as compared to control group. In EL and VC treated rats, urine volume was significantly reduced as compared to $\mathrm{GM}$ treated rats $(\mathrm{GM}+\mathrm{EL}$ and $\mathrm{GM}+\mathrm{VC}$ groups). In fact, EL and $\mathrm{VC}$ treated rats showed decrease in the urine volume to the level of the control.

Serum creatinine and urea: Serum creatinine level increased by $368 \%$ and Blood Urea Nitrogen level (BUN) increased by $176 \%$ in the GM group compared to the control (C) group (Table 2). EL attenuated the increase in creatinine by 60 and $63 \%$ fold in BUN level. Similarly, VC attenuated the increase in creatinine and BUN level by $55 \%$.

LPO, GSH and Antioxidant activity in mitochondrial and post-mitochondrial fraction of kidney; MDA is a marker of oxidative stress. GM treated group showed 159 and $123 \%$ increase in MDA levels of mitochondrial and post-mitochondrial fraction respectively. Thus the content of LPO is higher in mitochondrial fraction than in post-mitochondrial fraction (Fig. 1). The EL treated group showed 80 and $63 \%$ decrease in mitochondrial and post-mitochondrial MDA levels respectively. However, the VC treated group decreases MDA levels by 77 and $64 \%$ in mitochondrial and post-mitochondrial respectively. Decrease in MDA levels were more in rats treated with EL compared to VC treated rats.

The GSH content in the post-mitochondrial fraction was relatively higher as compared to mitochondrial fraction in control group. GM treated rats showed decrease in GSH by $50 \%$ in mitochondrial fraction and $33 \%$ in post-mitochondrial fraction (Fig. 2). The EL treated group showed 75 and $88 \%$ improvement in GSH content of mitochondrial and post-mitochondrial fractions respectively. Similarly, VC treated group showed 62 and $89 \%$ increase in mitochondrial and post-mitochondrial GSH content.

Table 1:Effect of EL treatment on GM-induced changes body weight and urine volume

\begin{tabular}{|c|c|c|}
\hline Groups & Body weight (gm sec) & Urine volume $\left(\mathrm{mL} 24 \mathrm{~h}^{-1}\right)$ \\
\hline & $272 \pm 8.33$ & $5.8 \pm 0.24$ \\
\hline GM & $267 \pm 6.63$ & $15.6 \pm 1.18^{\text {aaa }}$ \\
\hline $\mathrm{GM}+\mathrm{EL}$ & $270 \pm 8.22$ & $7.3 \pm 0.39^{\mathrm{bbb}}$ \\
\hline $\mathrm{GM}+\mathrm{VC}$ & $272 \pm 7.66$ & $6.9 \pm 0.25^{\mathrm{bbb}}$ \\
\hline
\end{tabular}

Values are expressed as mean \pm SEM ( $\mathrm{n}=6$ in each group). aaa, $\mathrm{p}<0.001 \mathrm{Vs}$ C, bbb, $\mathrm{p}<0.001$ Vs GM. $\mathrm{C}=$ Control: $\mathrm{GM}=$ Gentamicin treated $\mathrm{GM}+\mathrm{EL}=$ Getamicin $+\mathrm{EL}$ methanolic extract treated GM+VC $=$ Getamicin + Vitamin C treated

Table 2: Effect of EL treatment on GM-induced changes in creatinine, BUN and tubular necrosis

\begin{tabular}{lccc}
\hline & Cretonne $(\mathrm{mg} / \mathrm{dL})$ & BUN $(\mathrm{mg} / \mathrm{dL})$ & Tubular necrosis \\
\hline $\mathrm{C}$ & $0.76 \pm 0.04$ & $21.5 \pm 1.08$ & ---- \\
GM & $3.57 \pm 0.14^{\mathrm{aaa}}$ & $59.3 \pm 2.31^{\mathrm{aaa}}$ & +++ \\
GM+EL & $1.88 \pm 0.11^{\mathrm{bb}}$ & $35.3 \pm 2.06^{\mathrm{bb}}$ & ++ \\
GM+VC & $2.02 \pm 0.08^{\mathrm{bbb}}$ & $38.7 \pm 1.31^{\mathrm{bbb}}$ & ++ \\
\hline
\end{tabular}

Values are expressed as mean \pm SEM ( $\mathrm{n}=6$ in each group). aaa, p <0.001 Vs. C, bbb, p<0.001 Vs GM. C = Control GM = Gentamicin treated $\mathrm{GM}+\mathrm{EL}=$ Getamicin $+\mathrm{EL}$ methanolic extract treated $\mathrm{GM}+\mathrm{VC}=$ Getamicin + Vitamin $\mathrm{C}$ treated
Treatment with EL and VC were having similar effect on GSH content of mitochondrial and postmitochondrial fractions.

SOD activity was higher in post-mitochondrial fraction as compared to mitochondrial fraction in control rats. The activity of SOD decreases by $61 \%$ in mitochondrial fraction, while decreases by $36 \%$ in postmitochondrial fraction (Fig. 3). The EL treated group showed 78 and $66 \%$ increase in mitochondrial and postmitochondrial SOD activity, respectively. Whereas VC treated group showed 73 and $57 \%$ increase in mitochondrial and post-mitochondrial SOD activity. EL treated rats showed little bit more increase in postmitochondrial SOD activity as compared to VC treated rats, but having similar effect on mitochondrial SOD activity. GPx activity in the mitochondrial fraction was relatively higher than the post-mitochondrial fraction in control rats (Fig. 4). Mitochondrial GPx activity decreases by $60 \%$, while post-mitochondrial activity decreases to $43 \%$ in GM-treated rats. The EL treated group showed increase in GPx activity by 80 and $113 \%$ in mitochondrial and post-mitochondrial fraction respectively. However, the VC treated group showed 82 and $101 \%$ increase in mitochondrial and postmitochondrial GPx activity. Effect of EL and VC on GPx activity of mitochondrial and post-mitochondrial compartments was comparable. Catalase activity decreases $42 \%$ in post-mitochondrial fraction in GM treated group as compared to control group (Fig. 5). The EL treated group showed $95 \%$ increase in activity, while the VC treated group showed $93 \%$ increase in CAT activity. EL and VC have similar effect on postmitochondrial CAT activity.

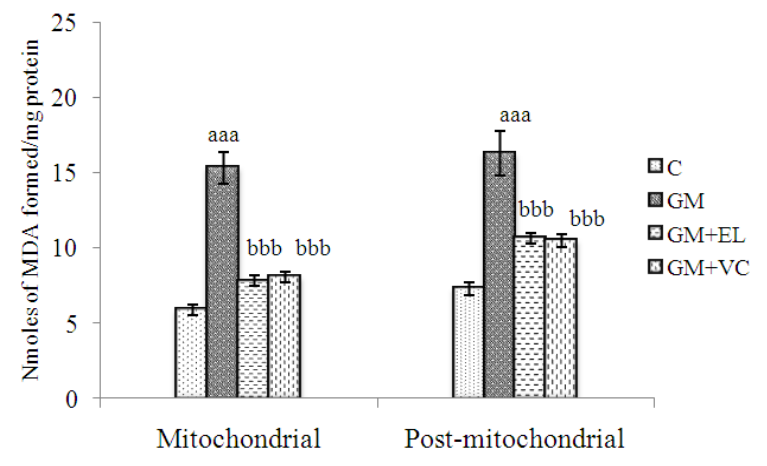

Fig. 1: Effect of EL on kidney lipid peroxidation levels in GM-induced nephrotoxicity (Values are expressed as mean \pm SEM $(n=6$ in each group). a, $\mathrm{p}<0.05$, aa, $\mathrm{p}<0.01$, aaa, $\mathrm{p}<0.001 \mathrm{Vs} \mathrm{C}, \mathrm{b}$, $\mathrm{p}<0.05$, bb $\mathrm{p}<0.01, \mathrm{bbb}, \mathrm{p}<0.001 \mathrm{Vs}$ GM) 


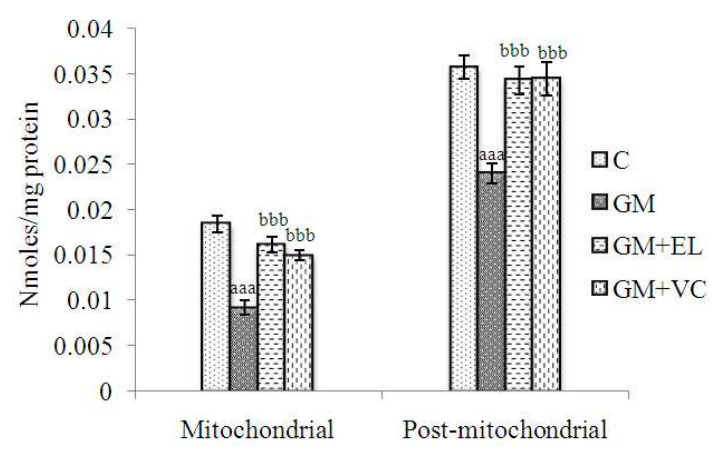

Fig. 2: Effect of EL on kidney reduced glutathione levels in GM-induced nephrotoxicity (Values are expressed as mean \pm SEM $(n=6$ in each group). a, $\mathrm{p}<0.05$, aa, $\mathrm{p}<0.01$, aaa, $\mathrm{p}<0.001$ vs. $\mathrm{C}, \mathrm{b}, \mathrm{p}<0.05, \mathrm{bb} \mathrm{p}<0.01, \mathrm{bbb}, \mathrm{p}<0.001 \mathrm{Vs} \mathrm{GM})$

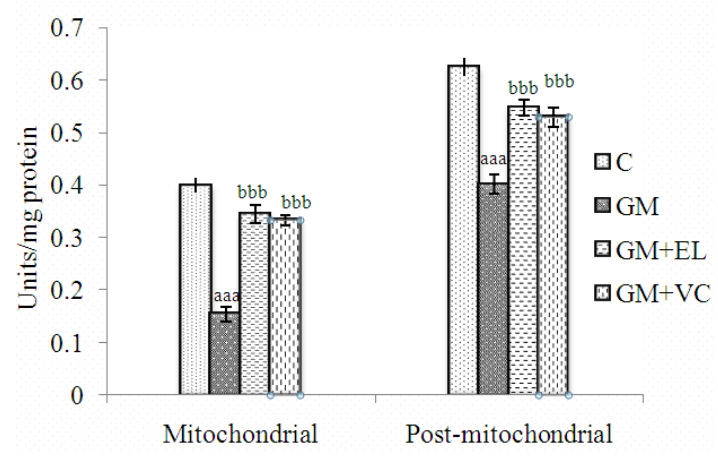

Fig. 3: Effect of EL on kidney superoxide dismutase activity in GM-induced nephrotoxicity Values are expressed as mean \pm SEM $(n=6$ in each group). a, $\mathrm{p}<0.05$, aa, $\mathrm{p}<0.01$, aaa, $\mathrm{p}<0.001 \mathrm{Vs}$ C, b, p<0.05, bb p <0.01, bbb, p <0.001 Vs GM

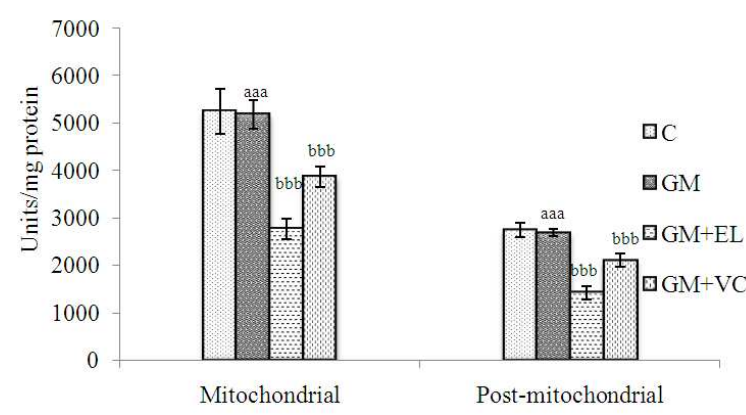

Fig. 4: Effect of EL on kidney glutathione peroxidase activity in GM-induced nephrotoxicity (Values are expressed as mean \pm SEM ( $\mathrm{n}=6$ in each group). a, $\mathrm{p}<0.05$, aa, $\mathrm{p}<0.01$, aaa, $\mathrm{p}<0.001 \mathrm{Vs}$ $\mathrm{C}, \mathrm{b}, \mathrm{p}<0.05$, bb $\mathrm{p}<0.01, \mathrm{bbb}, \mathrm{p}<0.001 \mathrm{Vs} \mathrm{GM})$

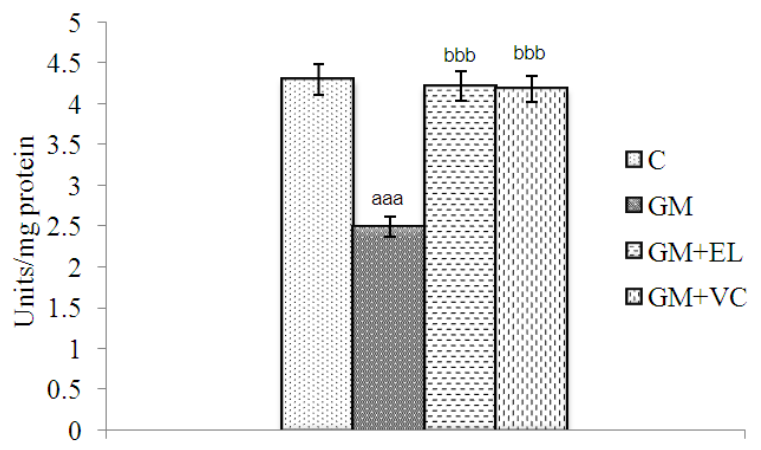

Fig. 5: Effect of EL on kidney catalase activity in GMinduced nephrotoxicity (Values are expressed as mean \pm SEM ( $n=6$ in each group). a, $\mathrm{p}<0.05$, aa, $\mathrm{p}<0.01$, aaa, $\mathrm{p}<0.001$ Vs $\mathrm{C}, \mathrm{b}$, $\mathrm{p}<0.05$, bb $\mathrm{p}<0.01$, bbb, $\mathrm{p}<0.001 \mathrm{Vs}$ GM, C = Control, $\mathrm{GM}=$ Gentamicin treated, $\mathrm{GM}+\mathrm{EL}=$ Getamicin+ EL methanolic extract treated, $\mathrm{GM}+\mathrm{VC}=$ Getamicin + Vitamin $\mathrm{C}$ treated)

Histopathological analysis: Rats treated with GM showed sever tubular necrosis (Table 2). Treatment with GM alone caused a marked vacuolization and necrosis $(+++)$ in proximal tubular epithelial cells. Treatment with EL (++) and VC (++) significantly decreases GM-induced tubular necrosis.

\section{DISCUSSION}

Previous studies with EL in our lab, has shown that methanolic extract of EL showed significant antioxidant activity in-vitro and in-vivo (Maroo et al., 2003b). As ROS play very important role in development of pathogenic condition of many diseases, like diabetic nephropathy. We hypothesized EL extract will protect rats from GM-induced nephrotoxicity, which is mainly caused by generation of ROS.

The results of the present study indicate that GM administration brought about a significant increase in BUN and serum creatinine levels, indicating kidney dysfunction. The results are in agreement with the reports of Kumar et al. (2000). These alterations in biochemical parameters were well correlated with the renal histological score of GM treated rats.

Reactive oxygen species including hydroxyl radical have been implicated in the etiology of GM gentamicin-induced nephrotoxicity. Walker and Shah (1988) showed that GM enhances the generation of hydrogen peroxide by renal cortical mitochondria and that iron chelators and hydroxyl-radical scavengers protect against GM-mediated renal damage. It has been also demonstrated generation of hydrogen peroxide by rat kidney cortex and glomeruli (Guidet and Shah, 
1989). Treatment with SOD or hydroxyl radical scavenger, Dimethyl-Thiourea (DMTU), two methods for reducing reactive oxygen metabolites, significantly lessened the GM-induced reduction in GFR (Nakajima et al., 1994). Cuzzocrea et al. (2002) demonstrate that daily administration of M40403, low molecular weight synthetic manganese containing superoxide dismutase mimetic, which selectively removes superoxide to gentamicin-treated rats, resulted in an almost complete normalization of kidney functions.

Our results show that GM-induced oxidative stress as demonstrated by significant elevation in lipid peroxidation and decrease in GPx and SOD activities both in mitochondrial and post-mitochondrial fraction. Lipid peroxidation levels were higher in mitochondrial fraction as compared to post-mitochondrial fraction. Similarly, decrease in mitochondrial SOD and GPx activity was higher in mitochondrial fraction as compared to post-mitochondrial fraction. The depletion in GSH content was higher in mitochondria fraction as compared to post-mitochondrial fraction. Decreased GSH status might be responsible for the observed decrease in GPx activity. Even post-mitochondrial CAT activity decreased significantly. Decrease in GPx and CAT activity indicates decrease efficiency in $\mathrm{H}_{2} \mathrm{O}_{2}$ scavenging activity in kidney tissue of GM treated rats, which might be responsible for increase in lipid peroxidation levels in both the fractions. Hence, above results clearly indicates higher oxidative stress in mitochondrial fraction as compared to postmitochondrial fraction in GM-induced nephrotoxicity. Histipathological changes observed in GM treated rat well correlates with GM-induced oxidative stress.

Oral administration of EL and Vit $\mathrm{C}$ was found to have a protective potential against GM-induced nephrotoxicity. Treatment with EL and Vit $\mathrm{C}$ showed increase in SOD and GPx activity, in both the fractions, along with increase in the GSH content, which is a primary antioxidant defense system of the cell. Amelioration in antioxidant defense system in both mitochondrial and post-mitochondrial fractions, leads to decrease in lipid peroxidation levels. However, the improvement in antioxidant defense system was more in mitochondrial fraction than in post-mitochondrial fraction which is a major site of ROS generation. In the present study, EL and Vit C treatment showed comparable protective effect on GM-induced nephrotoxicity. However, EL treatment showed better ameliorating effect on certain biochemical parameters as compared to Vit $\mathrm{C}$ treatment.

\section{CONCLUSION}

In summary, the present study provides evidence that co-administration of EL along with GM attenuates the increase in lipid peroxidative damage, restores antioxidant status, markers of renal injury and histopathological alterations. The present findings demonstrate that EL possesses significant therapeutic effects and it is a promising candidate for chemoprevention of GM-induced renal damage.

\section{ACKNOWLEDGMENTS}

I would like to thank Dr. Vihas Vasu for being resourceful person.

\section{REFERENCES}

Abdel-Gayoum, A.A., A.A. Bashir and M.M. ElFakhri, 1995. Effects of fish oil and sunflower oil supplementations on gentamicin-induced nephrotoxicity in rat. Human. Exp. Toxicol., 14: 884-888. DOI: 10.1177/096032719501401105

Abei, H., 1984. Catalase in vitro. Methods Enzymol., 105: 121-126. DOI: 10.1016/S00766879(84)05016-3 PMID: 6727660

Ali, B.H. and A.K. Bashir, 1996. Effect of superoxide dismutase treatment on gentamicin nephrotoxicity in rats. G. Pharmacol. Vascular Syst., 27: 349. DOI: 10.1016/0306-3623(95)02032-2

Ali, B.H., A.A. Al-Qarawi, O.M. Mahmoud and M. Hashad, 2004. Influence of spironolactone treatment on gentamicin-induced nephrotoxicity in rats. Basic. Clin. Pharmacol. Toxicol., 95: 20-23. PMID: 5245572

Ali, B.H., 2002. The effect of treatment with the medicinal plant Rhazya stricta decne on gentamicin nephrotoxicity in rats. Phytomedicine, 9: 385-389. DOI: 10.1078/09447110260571607

Al-Majed, A.A., A.M. Mostafa, A.C. Al-Rikabi and O.A. Al-Shabanah, 2002. Protective effects of oral arabic gum administration on gentamicin-induced nephrotoxicity in rats. Pharmacol. Res., 46: 445451. DOI: $10.1016 / \mathrm{S} 1043661802001251$

Babu, P.S. and P.S.M. Prince, 2004. Antihyperglycaemic and antioxidant effect of hyponidd, an ayurvedic herbomineral formulation in streptozotocin-induced diabetic rats. J. Pharm. Pharmacol., 56: 1435-1442. DOI: 10.1211/0022357044607

Baranisrinivasan, P., K.E. Elumalai, C. Sivakumar, V. Therasa and E. David, 2009. Hepatoprotective effect of Enicostemma littorale blume and Eclipta alba during ethanol induced oxidative stress in albino rats. Int. J. Pharmacol., 5: 268-272. 
Beuge, J.A. and S.D. Aust, 1978. Microsomal lipid peroxidation. Methods Enzymol., 52: 302-310. DOI: 10.1016/S0076-6879(78)52032-6

Beutler, E., O. Duron and B.M. Kelly, 1963. Improved method for the determination of blood glutathione. J. Lab. Clin. Med., 61: 882-888. PMID: 13967893

Bhatt, N., S. Barua and S. Gupta, 2009. Protective effect of enicostemma littorale blume on rat model of diabetic neuropathy. Am. J. Infect. Dis., 5: 99105. DOI: 10.3844/ajidsp.2009.99.105

Cuzzocrea, S., E. Mazzon, L. Dugo, I. Serraino and R.D. Paola et al., 2002. A role for superoxide in gentamicin-mediated nephropathy in rats. Eur. J. Pharmacol., 16: 67-76. DOI: 10.1016/S00142999(02)01749-1

Eisenberg, J.M., H. Koffer, H.A. Glick, M.L. Connell and L.E. Loss et al., 1987. What is the cost of nephrotoxicity associated with aminoglycosides? Ann. Int. Med., 107: 900-909. PMID: 3688681

Gopal, R., A. Gnanamani, R. Udayakumar and S. Sadulla, 2004. Enicostemma littorale blume-a potential hypolipidemic plant. Nat. Prod. Radi., 3: 401-405.

Guidet, B.R. and S.V. Shah, 1989. In vivo generation of hydrogen peroxide by rat kidney cortex and glomeruli. Am. J. Physiol., 256: F158-F164. PMID: 2912161

Gupta, R.S. and D. Singh, 2007. Hepatomodulatory role of Enicostemma littorale Blume against oxidative stress induced liver injury in rats. Afr. J. Agric. Res., 2: 131-138.

Gupta, S., N. Dadheech, A. Singh, S. Soni and R.R. Bhonde, 2010. Enicostemma littorale a new therapeutic target for islet neogenesis. Int. J. Integr. Biol., 9: 49-53.

Jaishree, V., S. Badami and S. Bhojraj, 2008. In vitro antioxidant activity of Enicostemma axillare. J. Health. Sci., 54: 524-528.

Karahan, I., A. Atessahin, S. Y1lmaz, A.O. Ceribas and F. Sakin, 2005. Protective effect of lycopene on gentamicin-induced oxidative stress and nephrotoxicity in rats. Toxicology, 215: 198-204. DOI: 10.1016/j.tox.2005.07.007

Kaushal, R., K.R. Dave and S.S. Katyare, 1999. Paracetamol hepatotoxicity and microsomal function. Environ. Toxicol. Pharmacol., 1: 67-74. DOI: 10.1016/S1382-6689(98)00053-2

Kavimani, S. and K.T. Manisenthlkumar, 2000. Effect of methanolic extract of Enicostemma littorale on Dalton's ascitic lymphoma. J. Ethnopharmacol., 71: 349-352. DOI: 10.1016/S0378-8741(00)00190-2
Kavutcu, M., O. Canbolat, S. Ozturk, E. Olcay and S. Ulutepe et al., 1996. Reduced enzymatic antioxidant defense mechanism in kidney tissues from gentamicin-treated guinea pigs: Effects of vitamins E and C. Nephron, 72: 269-274. DOI: 10.1159/000188853

Kumar, K.V., M.U.R. Naidu, A.A. Shifow and K.S. Ratnakar, 2000. Probucol protects against gentamicin-induced nephrotoxicity in rats. Ind. J. Pharmacol., 32: 108-113.

Lowry, O.H., N.J. Rosebrough, A.L. Farr and R.J. Randall, 1951. Protein measurement with the Folin phenol reagent. J. Biol. Chem., 193: 265-275. PMID: 14907713

Maldonado, P.D., D. Barrera, O.N. Medina-Campos, R. Hernandez-Pando and M.E. Ibarra-Rubio et al., 2003. Aged garlic extract attenuates gentamicin induced renal damage and oxidative stress in rats. Life Sci., 73: 2543-2556. DOI: 10.1016/S00243205(03)00609-X

Marklund, S. and G. Marklund, 1974. Involvement of the superoxide anion radical in the autoxidation of pyrogallol and a convenient assay for superoxide dismutase. Eur. J. Biochem., 47: 469-474. DOI: 10.1111/j.1432-1033.1974.tb03714.x

Maroo, J., A. Ghosh, R. Mathur, V.T. Vasu and S. Gupta, 2003a. Antidiabetic efficacy of Enicostemma littorale methanol extract in alloxaninduced diabetic rats. Pharm. Biol., 41: 388-391.

Maroo, J., V.T. Vasu and S. Gupta, 2003b. Dose dependent hypoglycemic effect of aqueous extract of Enicostemma littorale Blume in alloxan induced diabetic rats. Phytomed., 10: 196-199. DOI: 10.1078/094471103321659933

Maroo, J., V.T. Vasu, R. Aalinkeel and S. Gupta, 2002. Glucose lowering effect of aqueous extract of Enicostemma littorale Blume in diabetes: A possible mechanism of action. J. Ethnopharmacol., 81: 317-320. DOI: 10.1016/S0378-8741(02)00095-8

Mazzon, E., D., Britti, A.D. Sarro, A.P. Caputi and S. Cuzzocrea, 2001. Effect of $\mathrm{N}$-acetylcysteine on gentamicin-mediated nephropathy in rats. Eur. J. Pharmacol., 424: 75-83. DOI: 10.1016/S00142999(01)01130-X

Nakajima, T., A. Hishida and A. Kato, 1994. Mechanisms for protective effects of free radical scavengers on gentamicin-mediated nephropathy in rats. Am. J. Physiol., 266: F425-F431. PMID: 8160791 
Paglia, D.E. and W.N. Valentine, 1967. Studies on the quantitative and qualitative characterization of erythrocyte glutathione peroxidase. J. Lab. Clin. Med., 70: 158-169. PMID: 6066618

Patel, B.M. and S.M. Mishra, 2009. Aldose reductase inhibitory activity of a c-glycosidic flavonoid derived from enicostemma hyssopifolium. J. Complementary. Integrative. Med.. DOI: 10.2202/1553-3840.1217

Patel, S.P. and S.S. Katyare, 2006. Differential pH sensitivity of tissue superoxide dismutases. Ind. J. Clin. Biochem, 21: 129-133. DOI: 10.1007/BF02912927

Pedraza-Chaverri, J., A.E. Gonzalez-Orozco, P.D. Maldonado, D. Barrera and O.N. Medina-Campos et al., 2003. Diallyl disulfide ameliorates gentamicin-induced oxidative stress and nephropathy in rats. Eur. J. Pharmacol., 473: 71-78. DOI: 10.1016/S0014-2999(03)01948-4

Pedraza-Chaverri, J., P.D. Maldonado, O.N. MedinaCampos, I.M. Olivares-Corichi and M.D.L.A. Granados-Silvestre et al., 2000. Garlic ameliorates gentamicin nephrotoxicity: Relation to antioxidant enzymes. Free. Radical. Biol. Med., 29: 602-611. DOI: $10.1016 / \mathrm{S} 0891-5849(00) 00354-3$

Roy, S.P., C.M. Niranjan, T.M. Jyothi, M.M. Shankrayya and K.M. Vishawanath et al., 2010. Antiulcer and Anti-inflammatory Activity of Aerial Parts Enicostemma littorale Blume. J. Young. Pharm., 2: 369-373. DOI: 10.4103/09751483.71629

Sadique, J., T. Chandra, V. Thenmozhi and V. Elango, 1987. The anti-inflammatory activity of Enicostemma littorale and Mollugo cerviana. Biochem. Med. Metabolic. Biol., 37: 167-176. DOI: 10.1016/0885-4505(87)90023-5

Satav, J.G., K.R. Dave and S.S. Katyare, 2000. Influence of insulin status on extra-mitochondrial oxygen metabolism in the rat. Horm. Metab. Res., 32: 57-61. DOI: 10.1055/s-2007-978589

Sener, G., A.O. Sehirli, H.Z. Altunbas, Y. Ersoy and K. Paskaloglu et al., 2002. Melatonin protects against gentamicin-induced nephrotoxicity in rats. $\mathrm{J}$. Pineal. Res., 32: 231-236. DOI: 10.1034/j.1600079X.2002.01858.x

Sonawane, R.D., S.L. Vishwakarma, S. Lakshmi, M. Rajani and H. Padh et al., 2010. Amelioration of STZ-induced type 1 diabetic nephropathy by aqueous extract of Enicostemma littorale blume and swertiamarin in rats. Mol. Bio. Biochem., 340: 1-6. DOI: 10.1007/s11010-010-0393-x

Soni, S. and S. Gupta, 2009. In vitro Anti Plasmodial Activity of Enicostemma littorale. Am. J. Infect. Dis., 5: 259-262. DOI: 10.3844/ajidsp.2009.259.262
Srinivasan, M., M. Padmanabhan and P.S.M. Prince, 2005. Effect of aqueous Enicostemma littorale Blume extract on key carbohydrate metabolic enzymes, lipid peroxides and antioxidants in alloxan-induced diabetic rats. J. Pharm. Pharmacol., 57: 497-503. DOI: $10.1211 / 0022357055722$

Vaidya, H., M. Rajani, V. Sudarsanam, H. Padh and R. Goyal, 2009a. Antihyperlipidaemic activity of swertiamarin, a secoiridoid glycoside in poloxamer-407-induced hyperlipidaemic rats. J. Nat. Med., 63: 437-442. PMID: 19633811

Vaidya, H., M. Rajani, V. Sudarsanam, H. Padh and R. Goyal, 2009b. Swertiamarin: A lead from Enicostemma littorale Blume. for antihyperlipidaemic effect. Eur. J. Pharmacol. 617: 108-112. DOI: 10.1016/j.ejphar.2009.06.053

Vaijanathappa, J. and S. Badami, 2009. Antiedematogenic and Free Radical Scavenging Activity of Swertiamarin Isolated from Enicostemma axillare. Planta. Med., 75: 12-17. DOI: $10.1055 / \mathrm{s}-0028-1088333$

Vasu, V.T., C. Ashwinikumar, J. Maroo, S. Gupta and S. Gupta, 2003. Antidiabetic effect of enicostemma littorale Blume aqueous extract in newly diagnosed non-insulin-dependent diabetes mellitus patients (NIDDM): A preliminary investigation. Int. J. Pharm. Exp. Med., 3: 84-89. DOI: 10.3742/OPEM.2003.3.2.084

Vasu, V.T., H. Modi, J. Thaikoottathil and S. Gupta, 2005. Hypolipidaemic and antioxidant effect of Enicostemma littorale Blume aqueous extract in cholesterol fed rats. J. Ethnopharmacol., 101: 277282. DOI: $10.1016 /$ j.jep.2005.05.007

Vijayvargia, R., M. Kumar and S. Gupta, 2000. Hypoglycemic effect of aqueous extract of enicostemma littorale Blume (chhota chirayata) on alloxan induced diabetes mellitus in rats. Indian $\mathrm{J}$. Exp. Biol., 38: 781-784. PMID: 12557910

Walker, P.D., S.V. Shah, 1988. Evidence suggesting a role for hydroxyl radical in gentamicin-induced acute renal failure in rats. J. Clin. Investigat., 81: 334-341. DOI: $10.1172 / J C I 113325$

Walker, P.D., Y. Barri and S.V. Shah, 1999. Oxidant mechanisms in gentamicin nephrotoxicity. Renal. Failure., 21: 433-442. DOI: 10.3109/08860229909085109

Yang, C.L., X.H. Du and Y.X. Han, 1995. Renal cortical mitochondria are the source of oxygen free radicals enhanced by gentamicin. Renal Failure, 17: 21-26. DOI: 10.3109/08860229509036371 